\title{
Coordination between posture and phonation in vocal effort behavior
}

\author{
Commented by: Enio Lopes Mello ${ }^{1}$, Marta Assumpção de Andrada e Silva²
}

Lagier A, Vaugoyeau M, Ghio A, Legou T, Giovanni A, Assaiante C. Coordination between posture and phonation in vocal effort behavior. Folia Phoniatr Logop. 2010;62(4):195-202.

The study of Lagier et al. (2010) approaches a very important aspect of vocal production, which concerns the reciprocity of implications between postural adjustments and phonation. However, in spite of the importance of this issue, the authors reported in their literature review that there are few studies about this matter, even though it is widely approached in the clinical practice of speech therapy.

The authors based themselves on the assumption that the movement that is associated to vocal effort is structured, and involves the entire body. Thus, the head and other body parts have specific patterns of movement during phonation.

Based on this premise, Lagier and collaborators conducted a study which had the purpose of determining whether body movement is a collateral effect of vocal effort or a part of effort behaviors in communication.

For this purpose, the authors evaluated, simultaneously, the voice and posture of 20 speaking women, with an average of 26 years of age (interval: 20-43 years) with no history of laryngeal cancer, and without auditory disorders or postural issues. The subjects engaged in communication with a listener, aiming to be understood. They were asked to repeat 16 times, randomly, a series of numbers, short and informative words, with the purpose of increasing vocal effort in three different conditions:

- weak vocal effort (WVEC): subject and listener placed 4 meters (m) apart from each other, in a calm room with inside noise level varying in between 44 and $48 \mathrm{~dB}$;

- moderate vocal effort (MVEC): same conditions as above, but with speaker and listener placed $10 \mathrm{~m}$ apart;

- high vocal effort (HVEC): the distance between subject and listener was $10 \mathrm{~m}$, and both wore earphones playing a soundtrack composed by mixed sounds (reference: Phonak Party Night Noise), at an average of $90 \mathrm{~dB}$ SPL, combining reduced auditory feedback and Lombard effect.

While the listeners in front of the subjects wrote down what they did or did not understand, the speakers' speech

(1) Graduate Program (Doctorate level) in Speech-Language Pathology and Audiology, Pontifícia Universidade Católica de São Paulo - PUC-SP - São Paulo (SP), Brazil.

(2) Faculdade de Ciências Médicas da Santa Casa de São Paulo - FCMSCSP - São Paulo (SP), Brazil; Pontifícia Universidade Católica de São Paulo PUC-SP - São Paulo (SP), Brazil.

Correspondence address: Enio Lopes Mello. R. Dr. Alberto Seabra, 555, casa 4, Vila Madalena, São Paulo (SP), CEP 05452-000. E-mail: enio.mello@superig.com.br was recorded using the Evaluation voccale assitée (EVA)* equipment. For this purpose a microphone (AKG C 420) was positioned at $6 \mathrm{~cm}$ from the lip commissure, and vocal stimuli were registered using electroglotography (EGG). The vocal parameters assessed were: sound pressure level (SPL), length of utterance in milliseconds (ms), fundamental frequency $\left(\mathrm{f}_{0}\right)$ and glottal closure coefficient (GCC). For the purpose of postural assessment, 19 anatomical references were marked on the subjects bodies, using a pulse generator: middle forehead, temples, acromial processes, iliac spinal crests, lateral epicondyles, radial styloid processes, great trochanters, lateral condyles, lateral malleoli, and the heads of the fifth metatarsal. The movement data were collected using six TV cameras integrated with the SMART** smart system. Head, trunk and thigh positions in the sagittal plane were analyzed. Movement duration and amplitude were analyzed only during MVEC and HVEC.

The statistical analysis of the data used Friedman's ANOVA in order to test the global effect of the task, followed by Wilcoxon Signed Rank tests for comparison of pairs. The correlations were calculated and tested using Spearman's Coefficient, where the level of significance was established at $\mathrm{p} \leq 0.05$.

Significant results were found in between the three speech conditions for all vocal parameters - for SPL, average $\mathrm{f}_{0}, \mathrm{f}_{0}$ variation coefficient, for GCC and length of words in milliseconds. Vocal intensity, average $\mathrm{f}_{0}$ and length of utterance increased significantly from WVEC to MVEC, but there was no difference in the variation coefficient of $\mathrm{f}_{0}$ and closure coefficient. All parameters increased significantly from MVEC to HVEC: vocal intensity, average $\mathrm{f}_{0}$, length of utterance, variation coefficient of the fundamental frequency and closure quotient.

Regarding movement parameters, the amplitude and duration of backward movement of the head and trunk had a significant increase from MVEC to HVEC conditions, when

*EVA is a device which, attached to a computer, allows for recording and assessing simultaneously several vocal and word parameters, such as: fundamental frequency, intensity (sound pressure level), instability levels (jitter, shimmer, variation coefficient), spectral analysis, air volumes in breathing and speech, glottal resistance development. Available at: http://www.sqlab.fr/ (SQLab-LPL, Aix-en-Provence, France).

**SMART is a software for biomechanical analysis of movement, and is capable of integrating movement data synchronized with information derived from analogical devices, such as: strength platforms, electromyography (EMG) or other devices. It consists of infrared cameras, with $120 \mathrm{~Hz}$ frequency acquisition and a 768x576 pixels resolution, retromarker reflex device. 
compared to thigh movement. The beginning of the movement anticipated the beginning of phonation under MVEC (median: 313, 455 and $470 \mathrm{~ms}$ for the head, trunk and thighs, respectively) and HVEC (median: 424, 579 e $534 \mathrm{~ms}$ for head, trunk and thighs, respectively). The duration of the anticipation increased from MVEC to HVEC for the head and trunk, but not for the thighs. The correlation between movement amplitude and SPL was significant for 17/20 subjects for the trunk, and for $12 / 20$ subjects for the head.

The authors concluded that there was coordination between body posture and effort behavior. The movement associated to vocal effort is structured and involves the entire body. The amplitude and duration of movement increased simultaneously to the increase in vocal effort, and body movement anticipated phonation. The head and other body parts had specific movement patterns. Head movement may be involved with vocal efficiency, and the frontward flexion of the trunk may assure an energy gain in communication. Finally, the listener's perception of vocal effort may be an intrinsic part of the message.
Body movement and phonation are coordinated, and thus postural anticipation may be a resource to maintain the listener's attention on the content of the message.

We fully agree with the authors of the study on the strict relationship between body posture and vocal adjustments, since we understand the human body as a chain of muscles. These chains have different adjustments and muscled tensions for certain functions, as seen in phonation associated with posture. This process results in a balance between the different muscle chains, led by the action of agonist and antagonist muscles.

Even though the results and conclusions presented in the study are easy to understand and important to the field of voice in general, we would like to emphasize the innovative method presented by the researchers., showing a possibility of simultaneous assessment of voice and body, through the SMART system, and EVA which are still not widely used in Brazil, but which may aid in the understanding and eventual treatment of issues involving vocal adjustments and derivations of movements of postural balance and control. 